\title{
Inclusive prompt photon production in nuclear collisions at RHIC and LHC
}

\author{
François Arleo, ${ }^{a}$ Kari J. Eskola, ${ }^{b, c}$ Hannu Paukkunen, ${ }^{d}$ Carlos A. Salgado ${ }^{d}$ \\ ${ }^{a}$ Laboratoire d'Annecy-le-Vieux de Physique Théorique (LAPTH), \\ UMR5108, Université de Savoie, CNRS, \\ BP 110, 74941 Annecy-le-Vieux cedex, France \\ ${ }^{b}$ Department of Physics, University of Jyväskylä, \\ P.O. Box 35, FI-40014, Finland \\ ${ }^{c}$ Helsinki Institute of Physics, University of Helsinki, \\ P.O. Box 64, FIN-00014 Finland \\ ${ }^{d}$ Departamento de Física de Partículas and IGFAE, \\ Universidade de Santiago de Compostela, Santiago de Compostela, Spain \\ E-mail: arleo@lapp.in2p3.fr, kari.eskola@phys.jyu.fi, \\ hannu.paukkunen@usc.es, carlos.salgado@usc.es
}

ABSTRACT: Nuclear modification factors of inclusive prompt photon production in $d$-Au collisions at RHIC and $p$ - $\mathrm{Pb}$ collisions at the LHC are provided at different rapidities. The calculations are performed at NLO accuracy using the EPS09 NLO nuclear parton distribution functions (nPDFs) and their error sets. The results are compared to the ones obtained with the nDS and HKN07 NLO nPDFs, and to the corresponding nuclear modification factors of neutral pion production in these collisions. The sensitivity of these results to the scale choice is also investigated. Interestingly, the predictions using the different nPDF sets differ from each other to the extent that this observable can be expected to become very useful for probing nPDFs over a wide range of Bjorken- $x$. In order to obtain a perturbative QCD baseline in heavy-ion collisions, calculations are carried out for minimum bias $\mathrm{Au}-\mathrm{Au}$ collisions at $\mathrm{RHIC}$ and $\mathrm{Pb}-\mathrm{Pb}$ collisions at the LHC. We also estimate the maximal possible suppression which the produced QCD matter can be expected to have on inclusive prompt photon production due to the quenching of the fragmentation component. The nuclear modification factor for prompt photon production is thus suggested to be used for gauging both the cold and the hot nuclear matter effects on other hard processes which are expected to be affected by quark-gluon plasma formation, such as large- $p_{T}$ hadron and jet production.

KEYWORDS: QCD Phenomenology

ARXIV EPRINT: 1103.1471 


\section{Contents}

1 Introduction $\quad 1$

2 Theoretical framework $\quad 2$

2.1 Elements of the calculation 2

2.2 Nuclear parton distribution functions 3

3 Probing nPDFs in $p$ - $A$ collisions $\quad 4$

3.1 Feasibility of direct photon measurements 4

3.2 RHIC 6

3.3 LHC 8

$\begin{array}{llr}3.4 & \text { Scale dependence } & 9\end{array}$

4 Baseline pQCD predictions in heavy-ion collisions $\quad 11$

$\begin{array}{lll}4.1 & \text { Inclusive prompt photon production } & 11\end{array}$

4.2 Quenching of the fragmentation component 12

$\begin{array}{lll}5 & \text { Summary } & 13\end{array}$

\section{Introduction}

The production of inclusive and isolated prompt photons in hadronic collisions is a process that is known - along with jet production - to carry direct information on the gluon Parton Distributions Functions (PDF) in the proton [1, 2]. However, prompt photon production data are presently not used in the global fits of PDFs but rather provide an independent test of perturbative QCD and of the universality of the partonic densities. The good overall agreement between next-to-leading order (NLO) QCD calculations [3] and world data from fixed-target $(\sqrt{s}=23 \mathrm{GeV})$ to collider experiments $(\sqrt{s}=1.8 \mathrm{TeV})$ indicates that the underlying parton dynamics is properly understood. In this respect, the expected high-precision measurements to be performed at the LHC and RHIC might give extra constraints on the PDFs in the gluon sector [4]. The agreement between the first ATLAS and CMS measurements [5, 6] and NLO calculations is thus one more encouraging step towards this goal.

The recent global analyses of nuclear PDFs (nPDF) [7-14] have shown that nuclear collisions seem to follow the same collinear factorization theorem that works in hadronic collisions: Structure function data in nuclear (lepton) deep inelastic scattering (DIS) as well as Drell-Yan dilepton (DY) and hadron production measurements in high-energy proton(deuterium)-nucleus $(p(d)-A)$ collisions are consistent with universal (process independent) nuclear modifications of PDFs $[12,13]$. Other checks have been carried out 
recently using neutrino-DIS data $[15,16]$. Whether such a good description persists for prompt photon production has however not been verified yet, as no decisive-precision $p-A$ or $d-A$ data are presently available. Keeping in mind the ongoing efforts at RHIC and appreciating the potential capabilities of the LHC, this situation is expected to undergo a change in the near future.

As discussed in $[17,18]$, prompt photon production in $p$ - $A$ collisions appears to be a promising tool for probing the gluon nuclear densities, which up to now have been constrained through scaling violations of the nuclear DIS and DY data and inclusive $\pi^{0}$ production in $d$-Au collisions at RHIC [13] but, clearly, for which more constraints would be badly needed. Therefore, complementary to recent studies of inclusive pion production [19] and prompt photon+heavy quark production [20], we present and discuss in the present paper the NLO predictions for the nuclear modification ratios of prompt photon spectra in $d$-Au collisions at RHIC and $p$-Pb collisions at the LHC using modern nPDF sets (section 3). Baseline predictions in heavy-ion $(A-A)$ collisions will also be discussed in detail in section 4. Before this, let us first briefly look at the theoretical framework used in the present analysis.

\section{Theoretical framework}

\subsection{Elements of the calculation}

We consider in this analysis inclusive production of prompt photons,

$$
h_{1}+h_{2} \rightarrow \gamma+\mathrm{X}
$$

in $d$ - $\mathrm{Au}$ and $\mathrm{Au}-\mathrm{Au}$ collisions at RHIC $\left(\sqrt{s_{\mathrm{NN}}}=200 \mathrm{GeV}\right)$ as well as in $p$-Pb and $\mathrm{Pb}-\mathrm{Pb}$ collisions at the $\operatorname{LHC}\left(\sqrt{s_{\mathrm{NN}}}=8.8 \mathrm{TeV}\right.$ and $\sqrt{s_{\mathrm{NN}}}=5.5 \mathrm{TeV}$, respectively $)$ nominal energies.

Using collinear factorization, the inclusive photon production cross section can be written as a convolution

$$
d \sigma_{h_{1} h_{2} \rightarrow \gamma+X}=\sum_{i, j} f_{i}^{h_{1}}\left(M^{2}\right) \otimes f_{j}^{h_{2}}\left(M^{2}\right) \otimes d \hat{\sigma}_{i j \rightarrow \gamma+X^{\prime}}\left(\mu^{2}, M^{2}\right)+\mathcal{O}\left(1 / M^{2}\right),
$$

where $\otimes$ is an integral over the longitudinal momentum fractions $x_{1}$ and $x_{2}$ of the incoming partons $i$ and $j$, whose number densities - the PDFs of the projectile and target PDFs - are $f_{i}^{h_{1}}\left(x_{1}, M^{2}\right)$ and $f_{j}^{h_{2}}\left(x_{2}, M^{2}\right)$. The factorization scale entering the PDFs and the perturbatively calculable partonic pieces (subcross-sections at LO) $d \hat{\sigma}_{i j \rightarrow \gamma+X^{\prime}}$ is denoted by $M$, and the renormalization scale by $\mu$. As usual, $X$ indicates the inclusive nature of the cross section and $X^{\prime}$ indicates that in each partonic hard process (2-to-2 or 2-to-3) we integrate over everything else but the photon.

At leading order (LO) in pQCD, $\mathcal{O}\left(\alpha_{\mathrm{em}} \alpha_{s}\right)$, photons can be produced directly via two types of partonic subprocesses: Compton scattering $q(\bar{q}) g \rightarrow \gamma q(\bar{q})$ and annihilation $q \bar{q} \rightarrow \gamma g$. In terms of the transverse momentum $p_{T}$ and rapidity $y$ of the photon, and the cms-energy $\sqrt{s}$, the momentum fractions typically probed by direct photon production are $x_{1,2} \approx\left(2 p_{T} / \sqrt{s}\right) e^{ \pm y}$. Thus, towards smaller $p_{T}$, larger $y$ and larger $\sqrt{s}$, the process 
becomes sensitive to PDFs at smaller $x_{2}$ (target) and larger $x_{1}$ (projectile). At small values of $x$, the gluon distribution is larger than that of sea quarks, which is why the $q \bar{q}$ annihilation channel represents a small contribution to the cross sections in the entire kinematical domain reached at RHIC and LHC, see e.g. [4]. As a consequence, direct photon production is very sensitive to the gluon content of protons and nuclei at these colliders [17].

At next-to-leading order (NLO), $\mathcal{O}\left(\alpha_{\mathrm{em}} \alpha_{s}^{2}\right)$, there are altogether 7 types of contributing direct subprocesses (see [21]): $q(\bar{q}) g \rightarrow \gamma q(\bar{q}) g, q_{i} \overline{q_{i}} \rightarrow \gamma q_{i} \overline{q_{i}}, q_{i} \overline{q_{i}} \rightarrow \gamma q_{j \neq i} \overline{q_{j}}, q_{i} \overline{q_{i}} \rightarrow \gamma g g$, $q_{i}\left(\overline{q_{i}}\right) q_{j \neq i}\left(\overline{q_{j}}\right) \rightarrow \gamma q_{i}\left(\overline{q_{i}}\right) q_{j}\left(\overline{q_{j}}\right), q_{i}\left(\overline{q_{i}}\right) q_{i}\left(\overline{q_{i}}\right) \rightarrow \gamma q_{i}\left(\overline{q_{i}}\right) q_{i}\left(\overline{q_{i}}\right)$, and $g g \rightarrow \gamma q_{i} \overline{q_{i}}$. At LHC, $q g$ Compton scattering as well as gluon fusion are the processes which dominate the total cross section due to the high gluon density in the proton at small values of Bjorken- $x$.

In addition to the direct production channels discussed above, prompt photons can also be emitted through collinear fragmentation from high- $p_{T}$ quarks or gluons which are produced in primary hard partonic collisions. This is, again schematically, expressible as

$$
d \sigma_{h_{1} h_{2} \rightarrow \gamma+X}=\sum_{i, j} f_{i}^{h_{1}}\left(M^{2}\right) \otimes f_{j}^{h_{2}}\left(M^{2}\right) \otimes d \hat{\sigma}_{i j \rightarrow k+X^{\prime}}\left(\mu^{2}, M^{2}, M_{F}^{2}\right) \otimes D_{\gamma / k}\left(M_{F}^{2}\right) .
$$

The perturbatively calculable pieces related to the partonic hard processes, $d \hat{\sigma}_{i j \rightarrow k+X^{\prime}}$, are now of the order $\mathcal{O}\left(\alpha_{s}^{2+n}\right)$ ( $n=0$ for LO, and 1 for NLO parton production). The collinear divergences associated to this source of photons are resummed and absorbed into scaledependent fragmentation functions (FFs) into photons, $D_{\gamma / k}\left(z, M_{F}^{2}\right)$, where $z$ is the fractional momentum over which the last convolution is taken, and $M_{F}$ is the factorization scale related to the fragmentation process. The FFs scale asymptotically like $\mathcal{O}\left(\alpha_{\mathrm{em}} / \alpha_{s}\right)$ [22, 23], and thus the convolution of the FFs with the (higher-order) partonic cross sections makes the fragmentation contributions of the same order as the direct channel.

We present here results from a complete NLO calculation, which includes both the direct and the fragmentation processes at this order. The inclusive calculations performed here are based on the INCNLO-package $[23,24]$. We use the CTEQ6.6M NLO PDF sets for the free proton PDFs [25] and the Bourhis-Fontannaz-Guillet fragmentation functions of photons [26]. The renormalization and factorization scales we take to be equal and of the order of the photon transverse momentum, $\mu^{2}=M^{2}=M_{F}^{2}=a \times p_{T}$. To investigate the uncertainty related to the perturbative nature of the calculation presented, we however vary the constant $a$ between $1 / 2$ and 2. Finally, the prompt photon results are also contrasted to what is expected for inclusive single-pion production, using the AKK08 set [27] of fragmentation functions.

\subsection{Nuclear parton distribution functions}

We estimate the nuclear modifications of the photon spectra at RHIC and LHC using the recent NLO releases of nuclear PDFs: nDS [10], EPS09 [13] and HKN07 [11]. All these sets quantify the scale-dependent ratios between the PDF of a proton inside a nucleus, $f_{i}^{p / A}$, and that in the unbound proton, $f_{i}^{p}$,

$$
R_{i}^{A}\left(x, Q^{2}\right) \equiv \frac{f_{i}^{p / A}\left(x, Q^{2}\right)}{f_{i}^{p}\left(x, Q^{2}\right)} .
$$



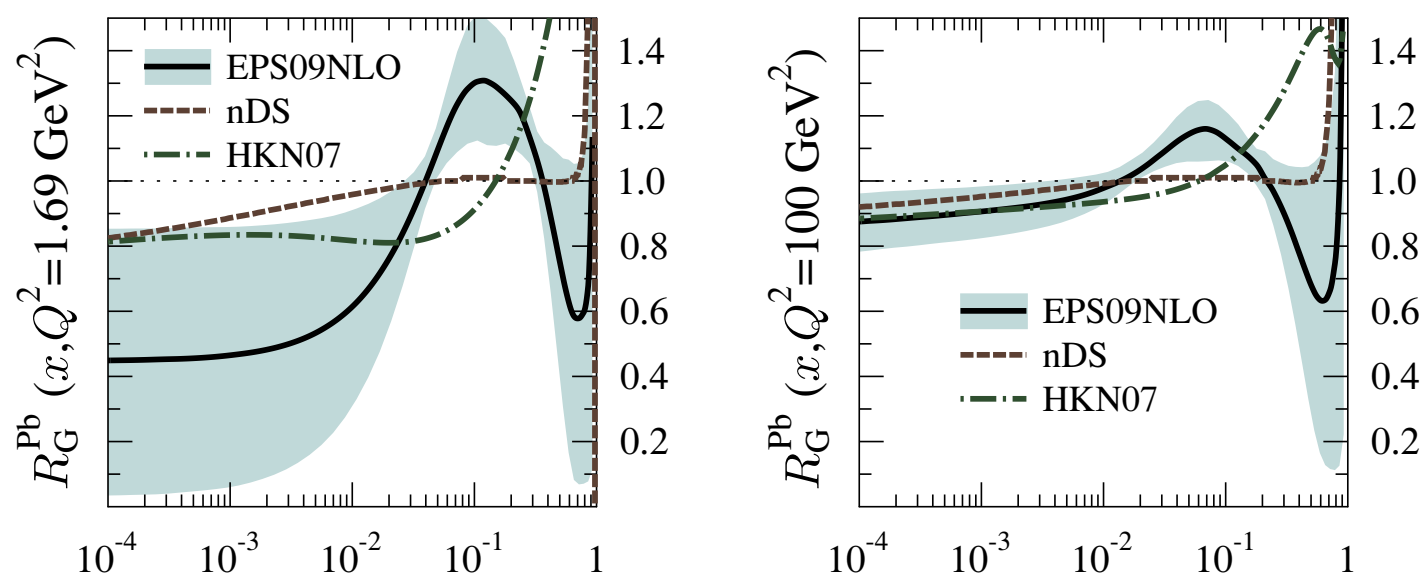

Figure 1. The nuclear modifications of the gluon PDF in a Pb-nucleus at $Q^{2}=1.69 \mathrm{GeV}^{2}$ (left) and $Q^{2}=100 \mathrm{GeV}^{2}$ (right) in the EPS09 set (and their uncertainties), nDS set and HKN set.

These modifications are known to exhibit a rich structure as a function of $x$. The suppression at small $x \lesssim 10^{-2}$ is commonly referred to as shadowing while an enhancement (anti-shadowing) is predicted around $x \sim 10^{-1}$. At $x \gtrsim 0.3$ the ratio becomes again smaller than 1 (EMC-effect), and larger than 1 just below $x=1$ because of the Fermimotion in nuclei.

The uncertainties related to these modifications are still significant especially in the low- $Q^{2}$ gluon sector, as shown in figure 1 (left panel) by the EPS09 uncertainty band on the ratio $R_{g}^{\mathrm{Pb}}$ at $Q^{2}=1.69 \mathrm{GeV}^{2}$. The small- $x$ uncertainties decrease, however, towards higher scales but the large- $x$ uncertainties remain, as is shown by the right panel of figure 1 . In the EPS09 set, the uncertainties of $R_{i}^{A}\left(x, Q^{2}\right)$ are encoded through 15 pairs of error sets, $S_{k=1,15}^{ \pm}$, whose gluon modifications are plotted as a light blue band in figure 1 . The propagation of the nPDF uncertainties into a physical quantity $X$, such as the gluon modification in figure 1 or prompt photon production considered here, can be obtained by squaring the deviations from the central result (which is obtained with the best fit, the set $S_{0}$ ) using the following prescription [13]:

$$
\begin{aligned}
& \left(\Delta X^{+}\right)^{2} \approx \sum_{i}\left[\max \left\{X\left(S_{i}^{+}\right)-X\left(S_{0}\right), X\left(S_{i}^{-}\right)-X\left(S_{0}\right), 0\right\}\right]^{2}, \\
& \left(\Delta X^{-}\right)^{2} \approx \sum_{i}\left[\max \left\{X\left(S_{0}\right)-X\left(S_{i}^{+}\right), X\left(S_{0}\right)-X\left(S_{i}^{-}\right), 0\right\}\right]^{2} .
\end{aligned}
$$

For more details about the nPDFs and their uncertainties, see ref. [13].

\section{Probing nPDFs in $p$ - $A$ collisions}

\subsection{Feasibility of direct photon measurements}

Before discussing the nPDF corrections to the prompt photon yield in nuclear collisions, we first briefly discuss the expected rates and hence the statistical accuracy at RHIC and 


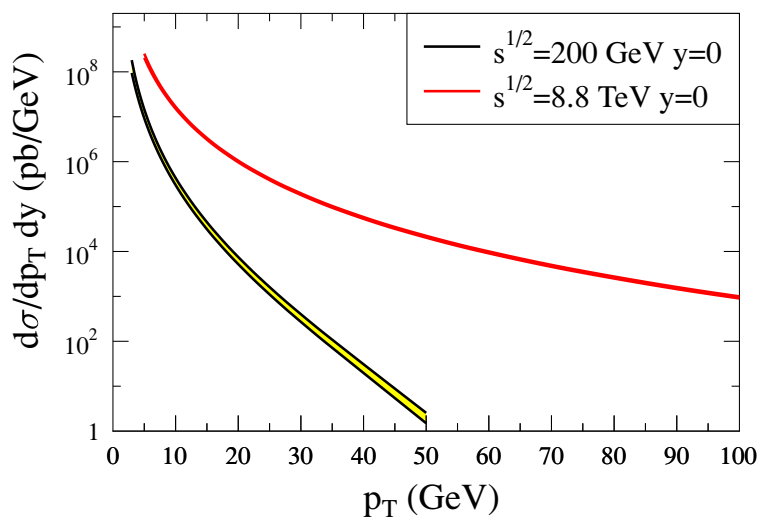

Figure 2. $d \sigma / d p_{T} d y$ inclusive photon production $p_{T}$-spectra in minimum-bias $d$-Au collisions at RHIC $\left(\sqrt{s_{\mathrm{NN}}}=200 \mathrm{GeV}\right)$ and $p$-Pb collisions at LHC $\left(\sqrt{s_{\mathrm{NN}}}=8.8 \mathrm{TeV}\right)$. The band (not visible at LHC energy) corresponds to a scale variation from $\mu=M=M_{F}=\left[p_{T} / 2 ; 2 p_{T}\right]$.

LHC. In figure 2 we plot the absolute NLO prompt photon $p_{T}$-spectra at mid-rapidity in (minimum-bias) $d$-Au collisions at RHIC and $p$-Pb collisions at the LHC, together with their scale uncertainty (band). Assuming that it would be necessary to reach a $\delta=10 \%$ statistical accuracy in order to measure meaningfully the nuclear effects in prompt photon production, one would need about $\mathcal{N}=\delta^{-2} \approx 100$ events in a $\left(p_{T}\right.$, rapidity)-bin.

The foreseen luminosity in $d$-Au collisions at RHIC-II is $\mathcal{L}^{\text {year }}=0.74 \mathrm{pb}^{-1}$ assuming 12 weeks of ion runs per year [28]. Requiring $\mathcal{N}=100$ events $/ \mathrm{GeV}$, the minimal cross section reads

$$
\frac{d \sigma^{\mathrm{dAu}}}{d p_{T} d y}=\frac{\mathcal{N}}{\mathcal{L}^{\text {int }}} \approx \frac{100}{0.74} \mathrm{pb} / \mathrm{GeV} \approx 1.4 \times 10^{2} \mathrm{pb} / \mathrm{GeV}
$$

which would be reached at $p_{T} \simeq 35 \mathrm{GeV}$, see figure 2 . At LHC such a statistical accuracy would correspond to a cross section

$$
\frac{d \sigma^{\mathrm{pPb}}}{d p_{T} d y}=\frac{100}{0.1} \mathrm{pb} / \mathrm{GeV} \approx 10^{3} \mathrm{pb} / \mathrm{GeV},
$$

assuming a rather conservative $\mathcal{L}=10^{29} \mathrm{~cm}^{-2} s^{-1}$ leading to a yearly integrated integrated luminosity ${ }^{1} \mathcal{L}^{\text {int }}=0.1 \mathrm{pb}^{-1}[29]$. From figure 2, this precision is achieved for transverse momenta up to $p_{T} \simeq 100 \mathrm{GeV}$ in one year. These considerations set the $p_{T}$-windows for the LHC and RHIC in which we perform our computations. Better precision could only be achieved of course in a more limited $p_{T}$ range. A more satisfactory $\delta=3 \%$ precision would be reached for transverse momenta less than $p_{T} \simeq 25 \mathrm{GeV}$ at RHIC and $p_{T} \simeq 60 \mathrm{GeV}$ at the LHC.

\footnotetext{
${ }^{1}$ We assume here that the LHC will run one month per year in the ion mode, which is taken to be $\Delta t \equiv 10^{6} \mathrm{~s}$ by convention.
} 

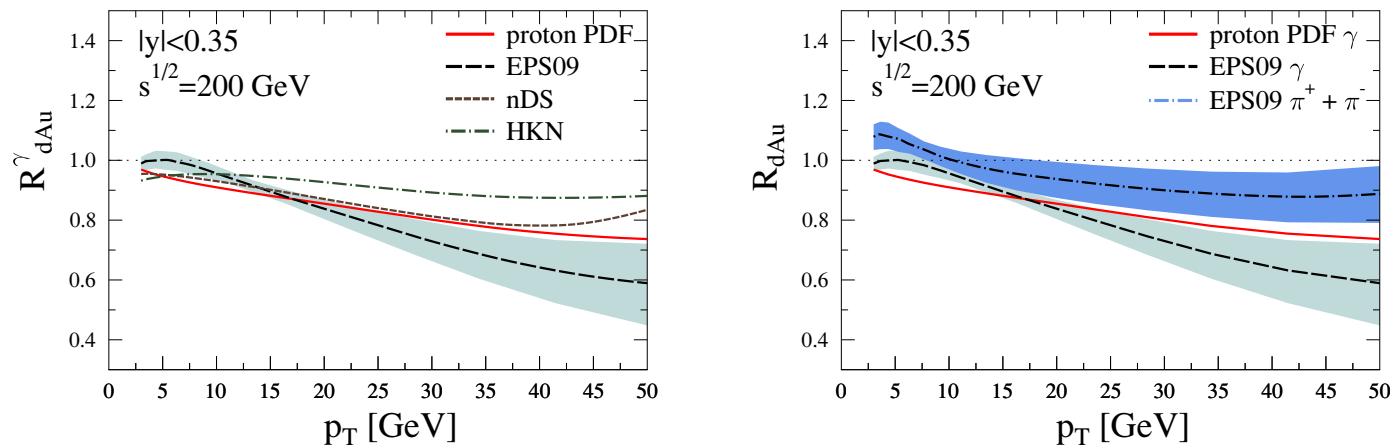

Figure 3. Nuclear modification ratio $R_{d \mathrm{Au}}^{\gamma}$ of inclusive photon production at $|y| \leq 0.35$ in $d$-Au collisions at $\sqrt{s_{\mathrm{NN}}}=200 \mathrm{GeV}$ using EPS09 nPDFs, in comparison to (i) nDS and HKN sets (left), and (ii) the pion case, $R_{d \mathrm{Au}}^{\pi}$ (right).

\subsection{RHIC}

Let us now move to the prediction of the nuclear modification ratio, or "quenching factor", in $d$-Au collisions at RHIC, defined as

$$
R_{d \mathrm{Au}}^{\gamma} \equiv \frac{d \sigma / d p_{T}(d+\mathrm{Au} \rightarrow \gamma+\mathrm{X})}{2 \times 197 \times d \sigma / d p_{T}(p+p \rightarrow \gamma+\mathrm{X})} .
$$

Note that the quenching factor (3.3) is not normalized to one when no nuclear modifications in the parton densities are assumed. The reason comes from an "isospin" effect as the density of up quarks - to which photons mostly couple - in the deuterium and the gold nucleus is smaller than that in a proton because of the presence of neutrons inside nuclei [30]. These corrections should be most pronounced whenever the valence quark sector of the nuclei is probed, that is at large $x_{1} / x_{2}$ and thus large $p_{T}$. On the contrary, no significant isospin effect is expected in charge-averaged or neutral hadron production which do not involve QED couplings.

The predictions for $R_{d \mathrm{Au}}^{\gamma}$ at mid-rapidity and $\sqrt{s_{\mathrm{NN}}}=200 \mathrm{GeV}$ are shown in figure 3 (left) in the transverse momentum range $p_{T}=5-50 \mathrm{GeV}$. The central EPS09 prediction is shown as a dashed black line, the light blue band corresponding to its uncertainty range, while the nDS (resp. HKN) prediction is denoted by the dotted brown (resp. dash-dotted green) curve. For separating the genuine nuclear effects from the sheer isospin effects, the solid red line indicates the calculation with no nuclear modifications in the PDFs (i.e. just the free proton PDFs are used) - it also serves as a comparison baseline for the nuclear effects obtained using the various $\mathrm{nPDF}$ sets.

The relative difference between EPS09 with respect to the baseline (red curve) prediction follows roughly the shape of the nuclear modifications in figure 1 . Below $p_{T} \lesssim 10 \mathrm{GeV}$ photon production is sensitive to anti-shadowing corrections $\left(x_{2} \sim 2 p_{T} / \sqrt{s_{\mathrm{NN}}} \simeq 0.1\right)$ while it is suppressed at larger momenta due to the EMC effect. This is also the case for pion production for which we only show the EPS09 prediction on $R_{d A u}^{\pi}$ as a blue band in the right panel of figure 3, except that in this case the suppression is practically free of isospin corrections, as mentioned above. 

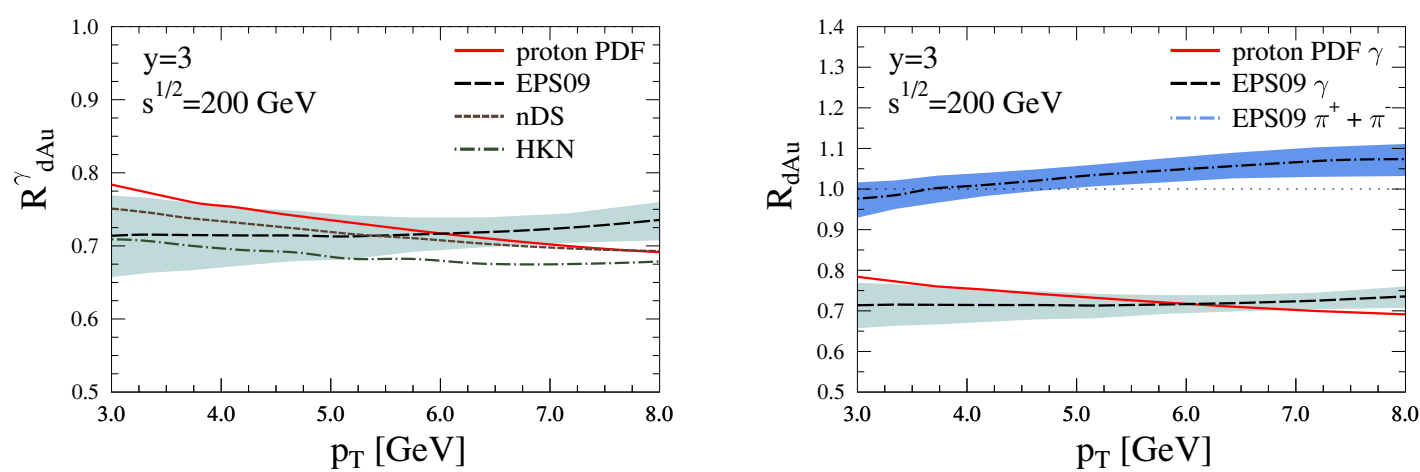

Figure 4. Same as figure 3 at $y=3$.

As noted in section 2.1, prompt photon production at forward rapidities, $y>0$, is more sensitive to lower momentum fractions in a target nucleus, $x_{2} \propto \exp (-y)$, than the mid-rapidity production, and therefore it probes efficiently the gluon nPDFs at small $x$. We demonstrate this in figure 4 (left) by plotting the analogue of figure 3 (left) at rapidity $y=3$ and in a narrower $p_{T}$-range due to phase-space restriction. As expected, $R_{d \mathrm{Au}}^{\gamma}$ at small $p_{T}$ falls now below the baseline (red) curve, signaling that this kinematical domain lies in the small- $x$ shadowing region $\left(p_{T}=5 \mathrm{GeV}\right.$ and $y=3$ corresponds to a typical value $\left.x_{2} \sim 2 p_{T} e^{-y} / \sqrt{s_{\mathrm{NN}}} \sim 2 \times 10^{-3}\right)$. From these figures, however, one may see that the predicted differences due to nPDF effects are not very large - of the order of $10 \%$ or less. For completeness, the quenching factor of single-pion production, $R_{d \mathrm{Au}}^{\pi}$, at $y=3$ is also plotted together with $R_{d \mathrm{Au}}^{\gamma}$ in figure 4 (right). The behaviour of $R_{d \mathrm{Au}}^{\pi}$ is again roughly similar to $R_{d \mathrm{Au}}^{\gamma}$ except for the isospin corrections.

It might be surprising at first glance to observe a strong isospin effect in the prompt photon channel at forward rapidity since quark distributions in a proton and in a neutron are symmetric at small values of $x: u^{p}(x)=d^{n}(x) \simeq d^{p}(x)=u^{n}(x)$. As a matter of fact, the strong isospin corrections visible in figure 4 actually come from the deuteron projectile which is probed at large $x_{1} \propto \exp (+y)$. As we shall see in the next section, unlike in the RHIC case, the isospin effect vanishes at forward rapidity at the LHC since the same projectile is used in $p-p$ and $p-\mathrm{Pb}$ collisions.

The RHIC photon data will be useful to learn about the behaviour of the gluon nPDFs from small to large $x$. In order to show the different kinematical regions probed at forward rapidities, $R_{d \mathrm{Au}}^{\gamma}$ is plotted in figure 5 (left) as a function of $p_{T}$ for three rapidity bins $(y=0, y=1.6$, and $y=2.4)$ which should be accessible by the PHENIX experiment at RHIC. The predictions are obtained assuming the EPS09 nPDFs. The EPS09 $R_{d A u}^{\gamma}$ ratio looks remarkably flat at all rapidity bins, even though the normalization differs: the larger the rapidity, the stronger the shadowing and the smaller the quenching factor. This flat behaviour is however somewhat accidental as it results from the interplay of isospin and nPDF effects. As can be seen in figure 5 (right) where the quenching factor is normalized with what is expected assuming isospin effects only, the behaviour is very different. ${ }^{2}$ At

\footnotetext{
${ }^{2}$ This would correspond roughly to the production ratio $\propto d^{3} \sigma(d+\mathrm{Au} \rightarrow \gamma+\mathrm{X}) / d^{3} \sigma(d+d \rightarrow \gamma+\mathrm{X})$ in which isospin corrections are very small.
} 

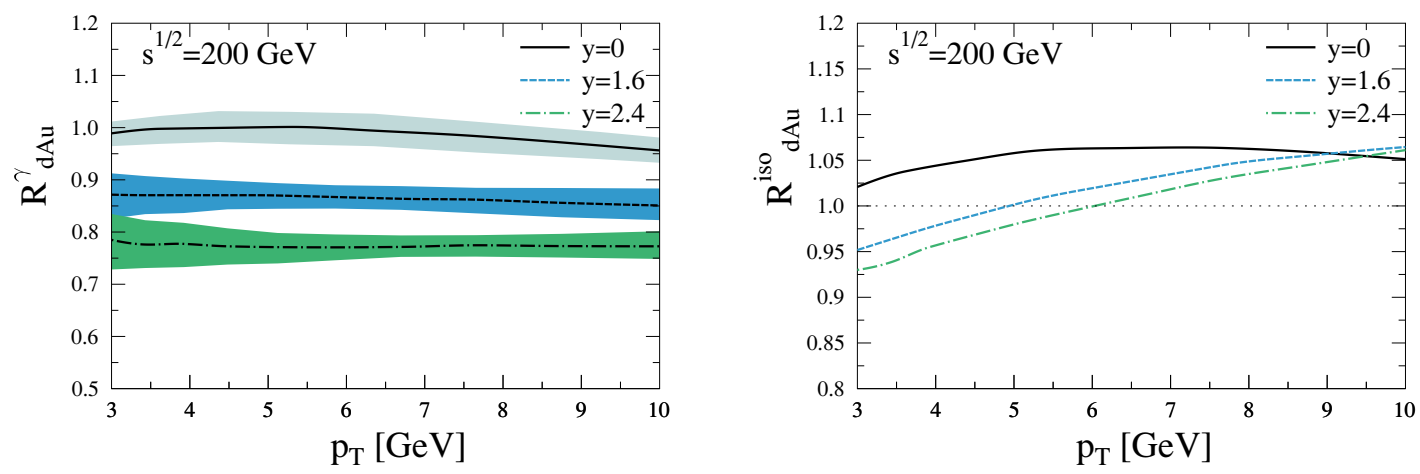

Figure 5. Left: Prompt photon quenching factor $R_{d \mathrm{Au}}^{\gamma}$ as a function of $p_{T}$ in different rapidities: $y=0$ (solid), $y=1.6$ (dotted) and $y=2.4$ (dash-dotted) with EPS09 nPDF corrections. Right: Double ratio $R_{d \mathrm{Au}}^{\text {iso }} \equiv R_{d \mathrm{Au}}^{\gamma}\left(\right.$ EPS09) $/ R_{d \mathrm{Au}}^{\gamma}$ (proton PDFs).

mid-rapidity, prompt photon production is sensitive to anti-shadowing effects in this $p_{T^{-}}$ range (see also figure 3 ) whereas the crossing region between anti-shadowing and shadowing is probed at $y=1.6$ and $y=2.4$ around $p_{T}=5-6 \mathrm{GeV}$.

\section{$3.3 \quad$ LHC}

In this section we give predictions for $p$-Pb collisions at the LHC. The nuclear modification factor,

$$
R_{p \mathrm{~Pb}}^{\gamma} \equiv \frac{d \sigma / d p_{T}(p+\mathrm{Pb} \rightarrow \gamma+\mathrm{X})}{208 \times d \sigma / d p_{T}(p+p \rightarrow \gamma+X)},
$$

is plotted for prompt photon production at mid-rapidity in figure 6 . Because of the larger center-of-mass energy, $\sqrt{s_{\mathrm{NN}}}=8800 \mathrm{GeV}$, the typical values of $x_{2} \propto 1 / \sqrt{s_{\mathrm{NN}}}$ probed in the nuclear target are much smaller than at RHIC. Remarkably, the differences in the results obtained with the different nPDF sets, are now more pronounced.

In the EPS09 set (light blue band), prompt photon production is enhanced with respect to the isospin effects (solid red line) above $p_{T}=20-40 \mathrm{GeV}$ due to the anti-shadowing. On the contrary, the transition from the shadowing to the anti-shadowing regions occurs only above $p_{T} \simeq 120 \mathrm{GeV}$ when using nDS (brown short-dashed) and HKN (green dot-dashed) $\mathrm{nPDF}$ sets. Like at RHIC, it is particularly interesting to note that the predictions using the latter two sets fall outside the EPS09 uncertainty band. As a consequence, this observable - which should be easily accessible at LHC - will allow one to set tighter constraints to the nuclear gluon densities. The EMC-effect (appearing at $x_{2} \gtrsim 0.1$ ) which can be probed easily at RHIC would become visible at the LHC only at very large $p_{T}, p_{T} \gtrsim 500 \mathrm{GeV}$ at $y=0$, or at negative rapidities, say, at $y<-2.2$ for $p_{T}=50 \mathrm{GeV}$.

The smallest $x_{2}$ values can be reached at LHC (because of the high c.m.s. energy), at forward rapidity and at not too large values of $p_{T}$. This kinematic region is therefore ideal for probing efficiently the shadowing region. This can be seen in figure 7 (left) where the photon suppression at $y=3$ is plotted as a function of $p_{T}$. As discussed in the previous section, no isospin corrections (solid red line) are expected since at small values of $x_{2}$ the 

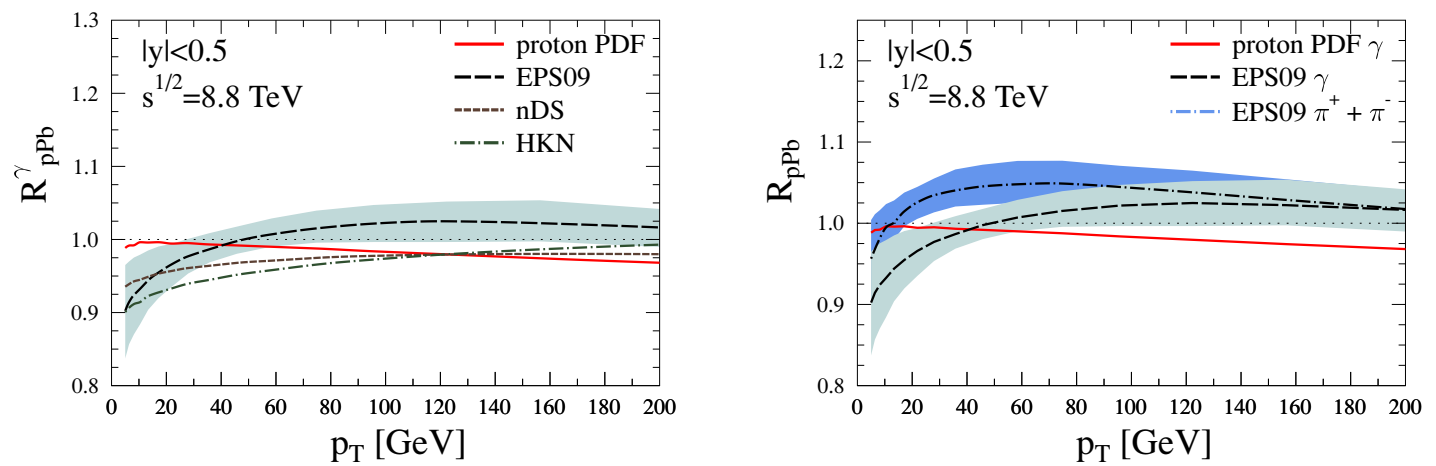

Figure 6. Nuclear modification ratio $R_{d \mathrm{Au}}^{\gamma}$ of inclusive photon production at $|y| \leq 0.5$ in $p$ - $\mathrm{Pb}$ collisions at $\sqrt{s_{\mathrm{NN}}}=8.8 \mathrm{TeV}$ using the EPS09 $\mathrm{nPDF}$ set, in comparison (left) to $\mathrm{nDS}$ and HKN $\mathrm{nPDF}$ and (right) to the pion production channel.

quark distributions in protons and neutrons are identical. Without a surprise, the photon quenching factor is below 1 because of the shadowing in all the nPDF sets studied here. The large uncertainty band of the EPS09 predictions in figure 7 reflects that of $R_{g}^{\mathrm{Pb}}$ shown in figure 1. Therefore, high-precision measurements at $p_{T} \simeq 10 \mathrm{GeV}$ and at $y=3$ in $p-\mathrm{Pb}$ collisions at LHC would bring significant further constraints for the nPDF global fit analyses.

Going to the backward direction at LHC would allow one to probe nPDFs in an $x$ region similar to that reached with mid-rapidity photon production at RHIC energy. As discussed in [17], measuring prompt photon production in $p$ - $A$ collisions at negative rapidity would allow one to access the quark nPDFs at large values of $x_{2}, R_{p \mathrm{~A}}^{y<0}\left(x_{\perp}\right) \simeq$ $R_{F_{2}}^{\mathrm{A}}\left(x_{\perp} \exp (-y)\right)$, while mid-rapidity photon production is sensitive to both the quark and gluon nPDFs, $R_{p \mathrm{~A}}^{y=0}\left(x_{\perp}\right) \simeq\left(\left(R_{F_{2}}^{\mathrm{A}}\left(x_{\perp}\right)+R_{G}^{\mathrm{A}}\left(x_{\perp}\right)\right) / 2\right.$. Figure 7 (right) shows the expected photon suppression in the rapidity bin $y=-3$ in the range $p_{T}=10-100 \mathrm{GeV}$. This range would correspond at RHIC, at similar values of $x_{2}$, to transverse momenta $p_{T}=\mathcal{O}\left(\sqrt{s_{\mathrm{NN}}} \mathrm{RHIC} / \sqrt{s_{\mathrm{NN}}} \mathrm{LHC} \times e^{3}\right)=5-50 \mathrm{GeV}$ at mid-rapidity. As can be seen from the comparison of figure 7 (right) with figure 3 (left), the expected photon suppression is rather similar. Remarkably, the spread of the EPS09 theoretical predictions proves narrower at the LHC than at RHIC, reflecting the fact that quark nPDFs are much better constrained than the gluon nPDFs at large values of $x[13] .^{3}$

\subsection{Scale dependence}

As discussed in section 2.1, collinearly factorized pQCD cross sections depend on the renormalization and factorization scales, which all are of the order of the photon $p_{T}$. In the absolute cross sections the sensitivity to these scales reflects the uncertainty which results from terminating the perturbation series at a certain order, and thus neglecting the higherorder corrections (here, NNLO and beyond). In the nuclear modification ratio $R_{d \mathrm{Au}}^{\gamma}$, such

\footnotetext{
${ }^{3}$ In addition, larger scales are probed at the LHC, $\left.Q^{2}\right|_{\text {LHC }} \sim 4 \times\left. Q^{2}\right|_{\text {RHIC }}$. This is however a rather moderate effect since the EPS09 gluon nPDF ratios do not exhibit a strong $Q^{2}$-dependence at large $x$ [13].
} 

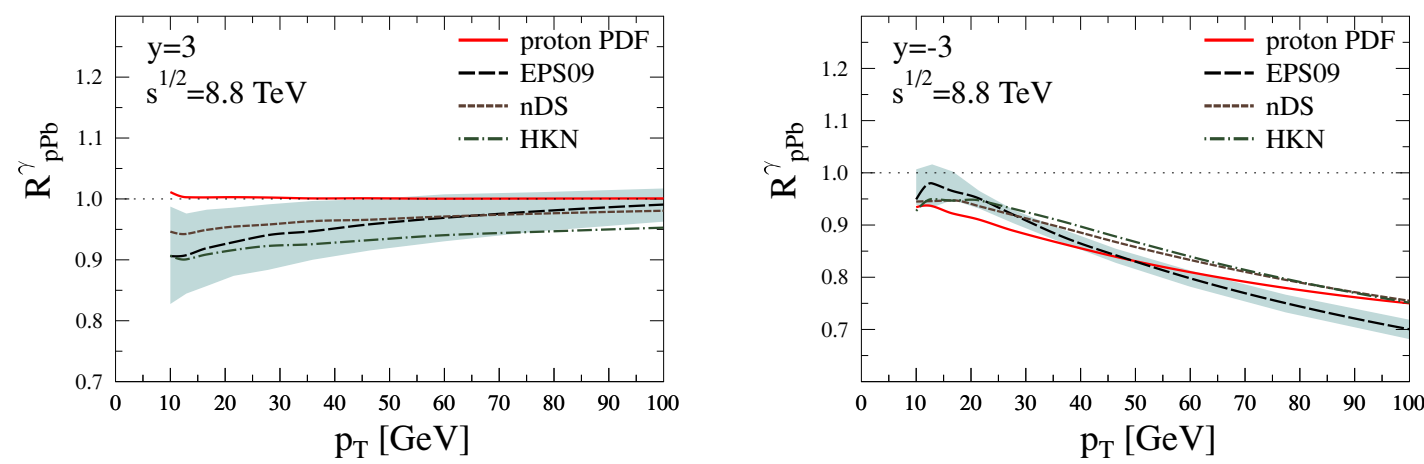

Figure 7. Nuclear production ratio $R_{d \mathrm{Au}}^{\gamma}$ of inclusive photon production at $y=3$ (left) and $y=-3$ (right) in $p$-Pb collisions at $\sqrt{s_{\mathrm{NN}}}=8.8 \mathrm{TeV}$ using the EPS09 nPDF set, in comparison to the nDS and HKN nPDF sets.

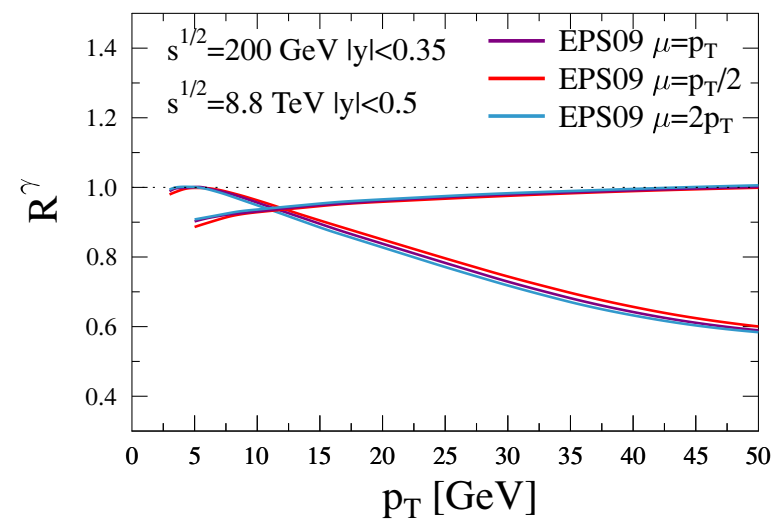

Figure 8. Scale dependence of the nuclear modification ratio $R_{d \mathrm{Au}}^{\gamma}$ for mid-rapidity prompt photon production at RHIC (lower curve) and LHC (upper curve). Calculations are performed using the central EPS09 nPDF set and varying all scales by a factor of two with respect to the photon transverse momentum; see the text for details.

scale uncertainties should nevertheless largely just cancel out. However, since the nPDF corrections $R_{i}^{A}\left(x, M^{2}\right.$ ) (eq. (2.3)) do depend on the factorization scale $M$, also $R_{d \mathrm{Au}}^{\gamma}$ may exhibit some dependence on $M$. To quantify this theoretical uncertainty, the nuclear modification ratio of prompt photon production at mid-rapidity has been computed using the EPS09 set and varying all scales, $\mu=M=M_{F}$ from $p_{T} / 2$ to $2 p_{T}$, as was done also in the calculation of the absolute cross section in section 3.1. As shown in figure 8 at the RHIC and LHC energies, the predictions show very little scale dependence. More importantly, the scale dependence proves much smaller than the current uncertainties in the nuclear modifications of the PDFs; see the band in figure 2 . 

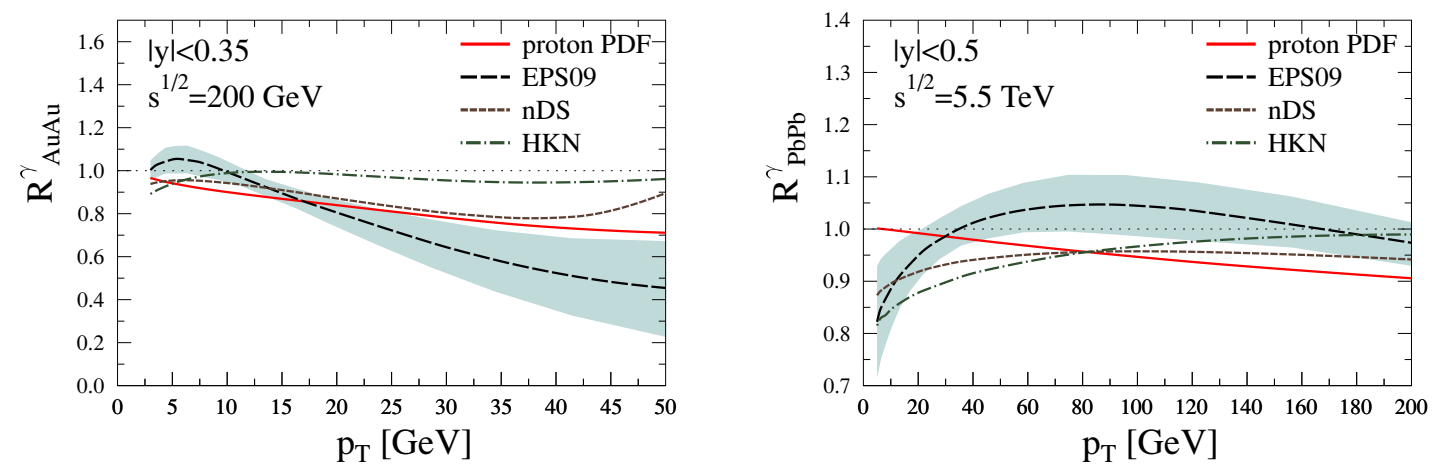

Figure 9. Left: Nuclear production ratio $R_{\mathrm{AuAu}}^{\gamma}$ of inclusive photon production in minimum-bias $\mathrm{Au}-\mathrm{Au}$ collisions at $\sqrt{s_{\mathrm{NN}}}=200 \mathrm{GeV}(|y| \leq 0.35)$ using EPS09, nDS and HKN nuclear parton densities. Right: same in $\mathrm{Pb}-\mathrm{Pb}$ collisions at $\sqrt{s_{\mathrm{NN}}}=5.5 \mathrm{TeV}(|y| \leq 0.5)$.

\section{Baseline pQCD predictions in heavy-ion collisions}

\subsection{Inclusive prompt photon production}

As can be seen in eqs. (2.1) and (2.2), nuclear corrections are expected to be much more pronounced in nucleus-nucleus collisions than in proton-nucleus collisions, very roughly $R_{A A} \sim R_{p A}^{2} \sim\left(R_{g}^{A}\right)^{2}$ in minimum-bias collisions. Therefore, it is particularly useful to investigate how prompt photon production in heavy-ion collisions would show the presence of a strongly interacting QCD-medium and possibly also constrain nuclear parton densities. This would also allow for more reliable estimates of other hard processes like jet and large$p_{T}$ hadron production, which, on the basis of RHIC data (see e.g. [31] for a review) and the first LHC measurements [32, 33], are generally believed to be affected by energy losses of hard partons in the formed quark-gluon plasma (QGP). Another motivation to make the baseline pQCD predictions for the $A$ - $A$ collisions here is that the $\mathrm{Pb}-\mathrm{Pb}$ programme at the LHC has already started.

The quenching factors in $\mathrm{Au}-\mathrm{Au}$ collisions at RHIC (left) and $\mathrm{Pb}-\mathrm{Pb}$ collisions at the LHC (right) are shown in figure 9. As expected, the shapes are similar but the nuclear effects are stronger than in the respective $p$ - $A$ collisions, as the comparison with figure 3 (left) and figure 6 (left) shows. Note moreover that the nPDF corrections might be even more pronounced in central collisions as compared to these minimum-bias predictions, which are averaged over all centralities. A study of the centrality dependence of $R_{\mathrm{AuAu}}^{\gamma}$ would require a modeling of the spatial dependences of the nPDFs, which is outside the scope of the present study. The PHENIX collaboration at RHIC has reported on high- $p_{T}$ measurements of $R_{\mathrm{AuAu}}^{\gamma}$ in central collisions [34] (see figure 2 there). However, since these data are still preliminary and since our present setup is consistent only with minimum-bias collisions, we do not make a detailed comparison with these data in this paper.

The plots in figure 9 thus set the stage for the conclusions to be made from the forthcoming data: agreement with the pQCD baseline in $p-A$ collisions but not in $A-A$ collisions would be a clear hint that photons are actually sensitive to hot medium effects. 

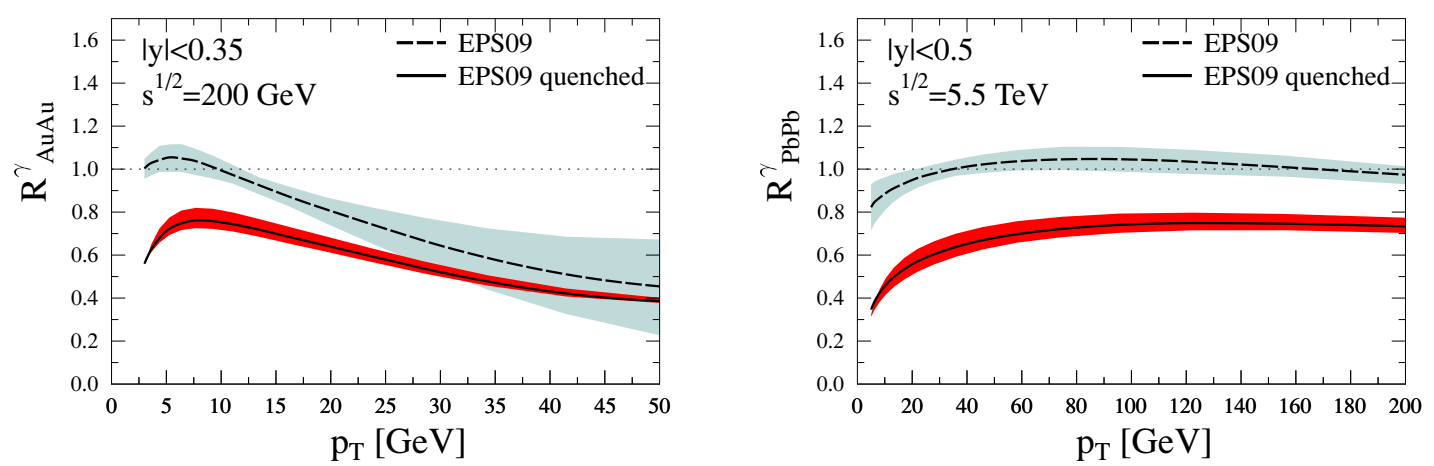

Figure 10. Upper bands: Same as figure 9 using the EPS09 nPDF sets. Lower bands: Nuclear production ratio $R_{A A}^{\gamma}$ using the central EPS09 nPDF set and after downscaling the fragmentation component by a factor of five. The band corresponds to a scale variation from $p_{T} / 2$ to $2 p_{T}$.

\subsection{Quenching of the fragmentation component}

It is not clear yet to what extent the production of prompt photons is affected by the presence of the dense medium in heavy-ion collisions. On the one hand, it has been suggested that the parton multiple scattering might enhance the photon yield either through jet-to-photon conversion [35] or medium-induced photon bremsstrahlung [36]. On the other hand, photon production might be suppressed due to the quenching of the fragmentation component as it happens for inclusive hadron production [30].

At large enough $p_{T}$, say at $p_{T} \gtrsim 5 \mathrm{GeV}$ at RHIC and possibly slightly above at LHC, one could nevertheless expect the suppression due to the quenching of the fragmentation component to be larger than the possible enhancement(s) caused by the medium. Therefore, we present in figure 10 a set of curves where we have by hand simply downscaled the fragmentation component by a factor of five (assuming in the calculation the central EPS09 set, for the illustration) which very roughly corresponds to the observed maximum suppression of large- $p_{T}$ hadrons at RHIC and LHC. These curves should therefore serve as a lower limit for prompt photon production in $A$ - $A$ collisions. It should, however, be noted that these additional curves are, strictly speaking, not physical quantities since the separation between direct and fragmentation components is ambiguous beyond the Born level and scale-dependent. As before, the calculations are performed for different scale choices from $p_{T} / 2$ to $2 p_{T}$ corresponding to the band in figure 10. We also reproduce in this figure the quenching factor obtained with the EPS09 nPDF sets (with its EPS09 uncertainty band) as shown in figure 9, i.e. without any rescaling of fragmentation component, for comparison.

The prompt photon suppression is most pronounced at small $p_{T}$, where the fragmentation component is the largest. As $p_{T}$ gets larger, the direct photon contribution becomes more important, and therefore the expected photon quenching weakens. At RHIC, the photon quenching becomes comparable to the prediction with no energy loss effects above $p_{T} \gtrsim 25 \mathrm{GeV}$. The RHIC predictions are qualitatively similar to the calculation performed in [30]. At LHC, the direct photon contribution to the total cross section is somewhat less than at RHIC. As a consequence, energy loss effects are more pronounced and might be visible in the entire $p_{T}$-range. 


\section{Summary}

Prompt photon production in $p$ - $A$ and $d-A$ collisions is among the best observables for probing parton distributions in nuclei, especially the nuclear gluon distributions, which are presently still poorly known. In this paper, we have computed the nuclear modification factors of single inclusive prompt photon production at NLO accuracy using the recent nPDF sets EPS09, nDS, and HKN. Calculations were carried out for $d$-Au collisions at RHIC and $p$ - $\mathrm{Pb}$ collisions at the LHC, for their nominal energies. The results are conveniently complementary: At mid-rapidities, prompt photon production at RHIC is mostly sensitive to the gluon anti-shadowing and the EMC effect, while at the LHC these photons probe the small- $x$ shadowing region. Complementary information on the gluon shadowing region can be obtained also from the forward-rapidity prompt photons both at RHIC and the LHC. Remarkably, by combining the future data at both colliders, one should be able to discriminate among the different existing sets of nPDFs.

On top of constraining the nuclear PDFs, inclusive prompt photon production can also be used as a "calibration measurement" in $A$ - $A$ collisions where QGP formation is expected to quench the production of large- $p_{T}$ partons, and thereby also the fragmentation component of prompt photon production. We have reported two pQCD baselines for this purpose: one by including the nuclear effects to the PDFs but no medium-effects to prompt photon production, and one by using the nPDFs and suppressing the fragmentation component by a factor of five.

The photons produced by medium-induced bremsstrahlung or through fragmentation processes are likely to be produced alongside a significant hadronic activity in their vicinity. This is also true - perhaps to a lesser extent - with the jet-photon conversion process, since the initial parton will radiate before "converting" into a photon though a rescattering in the QGP. Therefore, a way to reduce their contributions - and therefore medium effects - would be to trigger on isolated photons, i.e. using isolation criteria around each photon candidate. In heavy-ion collisions, the high-multiplicity would make this measurement extremely challenging, hopefully made possible using recent advances developed towards the reconstruction of jets in heavy-ion collisions [37]. Predictions of isolated photon production in nuclear collisions go beyond the scope of the present paper and will be presented elsewhere.

\section{Acknowledgments}

FA acknowledges the hospitality of the University of Jyväskylä where this work was initiated. KJE thanks Richard Seto from PHENIX for useful discussions and the Academy of Finland, Project nr. 133005, for financial support. This work is supported by Ministerio de Ciencia e Innovación of Spain under project FPA2009-06867-E; by Xunta de Galicia (Consellería de Educación and Consellería de Innovación e Industria - Programa Incite); by the Spanish Consolider-Ingenio 2010 Programme CPAN (CSD2007-00042); and by the European Commission grant PERG02-GA-2007-224770. CAS is a Ramón y Cajal researcher. 
Open Access. This article is distributed under the terms of the Creative Commons Attribution Noncommercial License which permits any noncommercial use, distribution, and reproduction in any medium, provided the original author(s) and source are credited.

\section{References}

[1] P. Aurenche, R. Baier, M. Fontannaz, J.F. Owens and M. Werlen, The gluon contents of the nucleon probed with real and virtual photons, Phys. Rev. D 39 (1989) 3275 [SPIRES].

[2] A.D. Martin, R.G. Roberts, W.J. Stirling and R.S. Thorne, Parton distributions: a new global analysis, Eur. Phys. J. C 4 (1998) 463 [hep-ph/9803445] [SPIRES].

[3] P. Aurenche, M. Fontannaz, J.-P. Guillet, E. Pilon and M. Werlen, A new critical study of photon production in hadronic collisions, Phys. Rev. D 73 (2006) 094007 [hep-ph/0602133] [SPIRES].

[4] R. Ichou and D. d'Enterria, Sensitivity of isolated photon production at TeV hadron colliders to the gluon distribution in the proton, Phys. Rev. D 82 (2010) 014015 [arXiv:1005.4529] [SPIRES].

[5] ATLAS collaboration, G. Aad et al., Measurement of the inclusive isolated prompt photon cross section in pp collisions at $\sqrt{s}=7 \mathrm{TeV}$ with the ATLAS detector, arXiv:1012.4389 [SPIRES].

[6] CMS collaboration, V. Khachatryan et al., Measurement of the isolated prompt photon production cross section in pp collisions at $\sqrt{s}=7$ TeV, Phys. Rev. Lett. 106 (2011) 082001 [arXiv: 1012.0799] [SPIRES].

[7] K.J. Eskola, V.J. Kolhinen and C.A. Salgado, The scale dependent nuclear effects in parton distributions for practical applications, Eur. Phys. J. C 9 (1999) 61 [hep-ph/9807297] [SPIRES].

[8] M. Hirai, S. Kumano and T.H. Nagai, Nuclear parton distribution functions and their uncertainties, Phys. Rev. C 70 (2004) 044905 [hep-ph/0404093] [SPIRES].

[9] M. Hirai, S. Kumano and M. Miyama, Determination of nuclear parton distributions, Phys. Rev. D 64 (2001) 034003 [hep-ph/0103208] [SPIRES].

[10] D. de Florian and R. Sassot, Nuclear parton distributions at next to leading order, Phys. Rev. D 69 (2004) 074028 [hep-ph/0311227] [SPIRES].

[11] M. Hirai, S. Kumano and T.H. Nagai, Determination of nuclear parton distribution functions and their uncertainties at next-to-leading order, Phys. Rev. C 76 (2007) 065207 [arXiv: 0709.3038] [SPIRES].

[12] K.J. Eskola, H. Paukkunen and C.A. Salgado, An improved global analysis of nuclear parton distribution functions including RHIC data, JHEP 07 (2008) 102 [arXiv:0802.0139] [SPIRES].

[13] K.J. Eskola, H. Paukkunen and C.A. Salgado, EPS09 - A new generation of NLO and LO nuclear parton distribution functions, JHEP 04 (2009) 065 [arXiv:0902.4154] [SPIRES].

[14] I. Schienbein et al., PDF nuclear corrections for charged and neutral current processes, Phys. Rev. D 80 (2009) 094004 [arXiv:0907.2357] [SPIRES].

[15] H. Paukkunen and C.A. Salgado, Compatibility of neutrino DIS data and global analyses of parton distribution functions, JHEP 07 (2010) 032 [arXiv: 1004.3140] [SPIRES]. 
[16] K. Kovarik et al., Nuclear corrections in neutrino-nucleus DIS and their compatibility with global NPDF analyses, Phys. Rev. Lett. 106 (2011) 122301 [arXiv:1012.0286] [SPIRES].

[17] F. Arleo and T. Gousset, Measuring gluon shadowing with prompt photons at RHIC and LHC, Phys. Lett. B 660 (2008) 181 [arXiv:0707.2944] [SPIRES].

[18] C. Brenner Mariotto and V.P. Goncalves, Nuclear shadowing and prompt photons in hadronic collisions at ultrarelativistic energies, Phys. Rev. C 78 (2008) 037901 [arXiv:0807.1680] [SPIRES].

[19] P. Quiroga-Arias, J.G. Milhano and U.A. Wiedemann, Testing nuclear parton distributions with pA collisions at the TeV scale, Phys. Rev. C 82 (2010) 034903 [arXiv:1002.2537] [SPIRES].

[20] T. Stavreva et al., Probing gluon and heavy-quark nuclear PDFs with gamma $+Q$ production in pA collisions, JHEP 01 (2011) 152 [arXiv: 1012.1178] [SPIRES].

[21] P. Aurenche, R. Baier, M. Fontannaz and D. Schiff, Prompt photon production at large $p_{T}$ scheme invariant $Q C D$ predictions and comparison with experiment, Nucl. Phys. B 297 (1988) 661 [SPIRES].

[22] J.F. Owens, Large momentum transfer production of direct photons, jets and particles, Rev. Mod. Phys. 59 (1987) 465 [SPIRES].

[23] P. Aurenche et al., A critical phenomenological study of inclusive photon production in hadronic collisions, Eur. Phys. J. C 9 (1999) 107 [hep-ph/9811382] [SPIRES].

[24] P. Aurenche, M. Fontannaz, J.P. Guillet, B.A. Kniehl and M. Werlen, Large $p_{T}$ inclusive $\pi_{0}$ cross-sections and next-to-leading-order QCD predictions, Eur. Phys. J. C 13 (2000) 347 [hep-ph/9910252] [SPIRES].

[25] P.M. Nadolsky et al., Implications of CTEQ global analysis for collider observables, Phys. Rev. D 78 (2008) 013004 [arXiv:0802.0007] [SPIRES].

[26] L. Bourhis, M. Fontannaz and J.P. Guillet, Quark and gluon fragmentation functions into photons, Eur. Phys. J. C 2 (1998) 529 [hep-ph/9704447] [SPIRES].

[27] S. Albino, B.A. Kniehl and G. Kramer, AKK update: improvements from new theoretical input and experimental data, Nucl. Phys. B 803 (2008) 42 [arXiv:0803.2768] [SPIRES].

[28] A.D. Frawley, F. Karsch, T. Ullrich and R. Vogt, Heavy Flavor, http://rhicii-science.bnl.gov/heavy/ (2006).

[29] A. Accardi et al., Hard probes in heavy ion collisions at the LHC: PDFS, shadowing and $p A$ collisions, hep-ph/0308248 [SPIRES].

[30] F. Arleo, Hard pion and prompt photon at RHIC, from single to double inclusive production, JHEP 09 (2006) 015 [hep-ph/0601075] [SPIRES].

[31] D. d'Enterria, Jet quenching, arXiv:0902.2011 [SPIRES].

[32] ALICE collaboration, K. Aamodt et al., Suppression of charged particle production at large transverse momentum in central Pb-Pb collisions at $\sqrt{s_{N N}}=2.76 \mathrm{TeV}$, Phys. Lett. B 696 (2011) 30 [arXiv:1012.1004] [SPIRES].

[33] ATLAS collaboration, G. Aad et al., Observation of a centrality-dependent dijet asymmetry in lead-lead collisions at $\sqrt{s_{N N}}=2.76 \mathrm{TeV}$ with the ATLAS Detector at the LHC, Phys. Rev. Lett. 105 (2010) 252303 [arXiv:1011.6182] [SPIRES]. 
[34] PHENIX collaboration, T. Isobe, Systematic study of high- $p_{T}$ direct photon production with the PHENIX experiment at RHIC, J. Phys. G 34 (2007) S1015 [nucl-ex/0701040] [SPIRES].

[35] R.J. Fries, B. Müller and D.K. Srivastava, High energy photons from passage of jets through quark gluon plasma, Phys. Rev. Lett. 90 (2003) 132301 [nucl-th/0208001] [SPIRES].

[36] B.G. Zakharov, Induced photon emission from quark jets in ultrarelativistic heavy-ion collisions, JETP Lett. 80 (2004) 1 [hep-ph/0405101] [SPIRES].

[37] M. Cacciari, J. Rojo, G.P. Salam and G. Soyez, Jet reconstruction in heavy ion collisions, Eur. Phys. J. C 71 (2011) 1539 [arXiv:1010.1759] [SPIRES]. 